\title{
Proposal of A Power-saving Unmanned Aerial Vehicle
}

\author{
Huimin $\mathrm{Lu}^{1, *}$, Yujie $\mathrm{Li}^{2}$, Joze Guna ${ }^{3}$, and Seiichi Serikawa ${ }^{1}$ \\ ${ }^{1}$ Kyushu Institute of Technology, Japan \\ luhuimindieee.org \\ ${ }^{2}$ Yangzhou University, China \\ ${ }^{3}$ University of Ljubljana, Slovenia
}

\begin{abstract}
In recent years, unmanned aerial vehicle (UAV) technologies are developing rapidly. Drone, one type of the UAVs, is used in many industrial fields, such as photography, delivery and agriculture. However, the commercial drone can flying only about 20 minutes at one charge. Furthermore, the drone prohibits flying at the limited area, and it also can't work in bad weather. Due to the development of drone technologies, we must reduce energy consumption, and realize high range movement. In order to solve these limitations, we develop a new type of drone, which has the function of flight and vehicle can move less power consumption. It extends high range of mobility to drone. Moreover, it can be used to pass through the limitation area or bad weather condition by sliding.
\end{abstract}

Keywords: Energy-saving, Drone, Energy.

\section{Introduction}

Because of faster control signal, smaller motor and lighter computer, the drone technology has developed rapidly. The drones have potential to be used in the fields of photography, delivery and agriculture, and around the world countries and companies have other potential applications. The BI intelligence expects that drone market is going to be reached at the $\$ 12$ billion until 2024 in the worldwide [1].

One the other hand, Japan doesn't have much large scale land compared with the other countries. It is possible that we can get benefit expanding the use of airspace. At the public-private Association for environment improvement of small unmanned air vehicle, we are aiming to realize delivering service by drone till 2019. In addition, aiming to realize a society of that autonomous drones are active in areas including cities from the 2020s [2]. An experiment was test on November, 2016 at the Chiba city which is the national strategic zone drone flied 700 meters over the sea and the book were reached at goal [3].

It is necessary that investment for technology by companies due to expand drones' use, but there are some restrictions. The following three points are representative.

1. The drone flies only $20 \sim 30 \mathrm{~min}$ and cannot go far away.

2. The drone is required circumvent around the no-fly zone.

3. The drone isn't safe in the strong wind.

As a conclusion, we need to develop drone that flying long distance and more efficiently [4]. 


\subsection{Purpose}

There are many previous studies for energy-saving of drones. First one is that car has only wheels and does not have motor but it move with wind power by propellers. However, this system has a drawback that it cannot move minutely. One of the benefit of flight and vehicle is that change position in detail, but it is so difficult to do that by wind power. The second one is that has crawler instead of wheels. However, crawler system cost high energy [5]. It consume about $11.1 \mathrm{~W}$ when it operations.

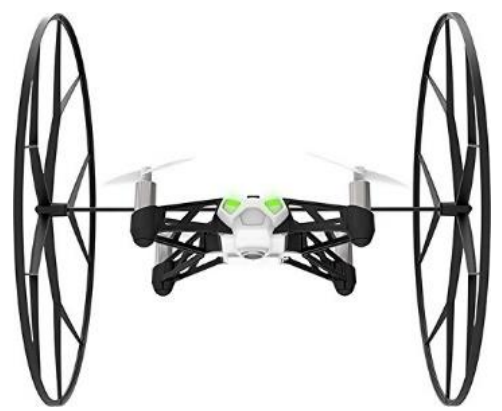

Fig. 1. Wind-type drone.

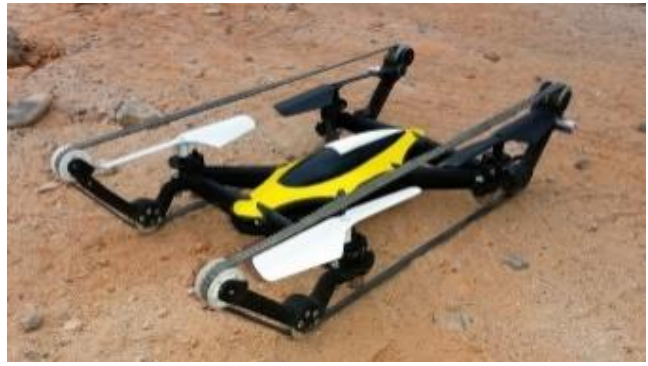

Fig. 2. Crawler-type drone.

We further to develop the drone with a motor car, and reduce power consumption. Furthermore, we compensate the shortcoming of flying by driving system. The purposes of our system are list in the following. (1) It realizes power saving by moving in the tire running, and it enables to deliver goods further away, and shooting of more distant situation than in the past; (2) By running in the no-fly zone, it is possible to pass through the area without detouring; (3) It allows to move on the ground without stopping even in the strong wind and to keep steady progress.

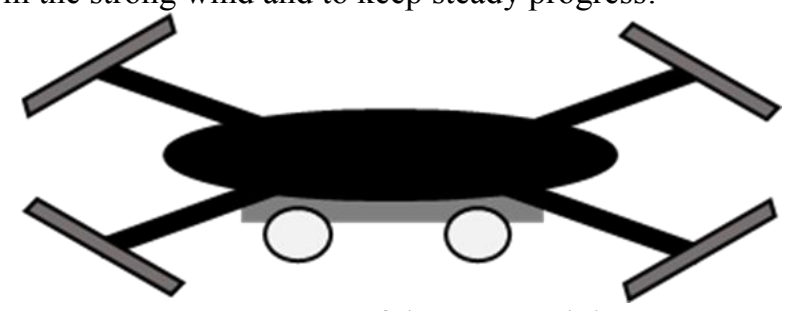

Fig. 3. Concept of the proposed drone. 


\section{Element of the System}

In this section, we explain these elements in details. The "Drone" meaning all kind of Unmanned Aerial Vehicle (UAV). In the UAVs, We use the quadcopter which has four propellers and we call it drone in this paper. Table. 1 summarizes the types of multicopters and there properties. In concrete, we use "Parrot AR.Drone 2.0" [6] in Figure. 4.

Table. 1. Types and properties of multicopter

\begin{tabular}{|c|c|c|c|c|}
\hline Type & Number of Rotors & Stability & Weight & Maintenance point \\
\hline Quadcopter & 4 & Low & Small & Few \\
\hline Hexacopter & 6 & $\mid$ & $\mid$ & | \\
\hline Octocopter & 8 & High & Large & A lot \\
\hline
\end{tabular}

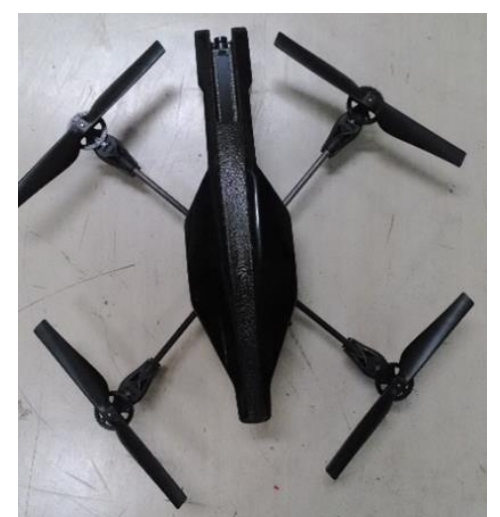

Fig. 4. Parrot AR.Drone 2.0.

In this system, we equip a tire type motor car to the lower part of the drone. The tire type car to be used is shown in Figure.5. In this research, we use FA-130 size motor, and the power is transmitted to the front and rear axles by a combination of the gear and the shaft.

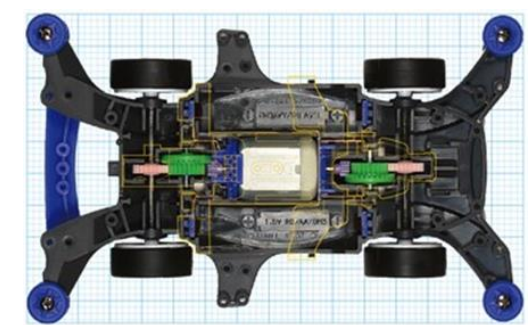

Fig. 5. Tire Type Vehicle

The used motor driver is Toshiba's TA7291P shown in Figure 6. This motor driver has ten terminals and it is used for controlling the DC motor. A large current is required to move the DC motor. Since the Raspberry Pi's GPIO has a limitation on the current, it is necessary to prevent flowing a large current on the GPIO. Therefore, using a motor 
driver makes it possible to control without directly connecting the DC motor to the GPIO.

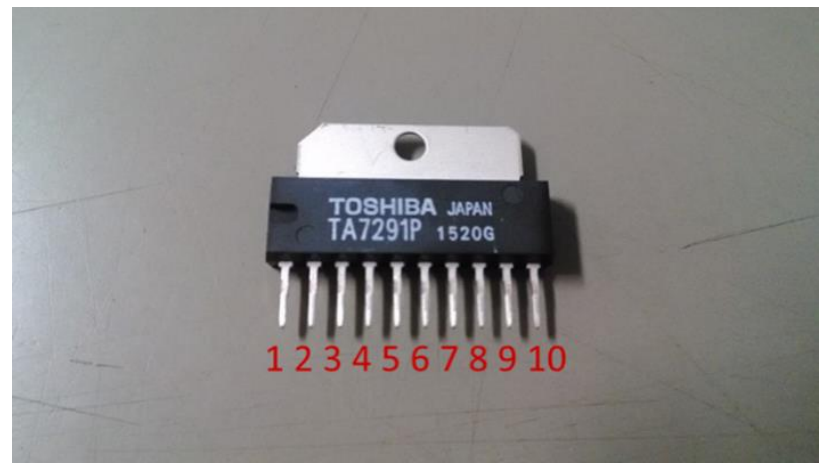

Fig. 6. Motor driver.

Table. 2. Motor driver terminals

\begin{tabular}{|c|c|c|}
\hline Pin Name & Pin Number & Use \\
\hline $\mathrm{V}_{\mathrm{CC}}$ & 7 & Power terminal of driver \\
\hline $\mathrm{V}_{\mathrm{S}}$ & 8 & Power terminal of motor \\
\hline $\mathrm{V}_{\text {ref }}$ & 4 & Iround \\
\hline $\mathrm{GND}$ & 1 & Imput terminal 1 \\
\hline IN1 & 5 & Motor control signal output terminal 1 \\
\hline IN2 & 6 & Motor control signal output terminal 2 \\
\hline OUT1 & 2 & $*$ 3pin and 9pin are NC terminal \\
\hline OUT2 & 10 & \multicolumn{2}{|c}{} \\
\hline \multicolumn{2}{|r}{}
\end{tabular}

We explain about each terminals in Table 2. First, we focus on two input terminals "IN 1", "IN 2" and two output terminals, "OUT 1", "OUT 2". "IN 1" and "IN 2" are connected to the two terminals of the raspberry pie GPIO, and "OUT 1" and "OUT 2" are connected to the two terminals of the DC motor.

Table. 3. Control by motor driver

\begin{tabular}{|c|c|c|}
\hline IN1 & IN2 & Motor behavior \\
\hline LOW & HIGH & Stop \\
\hline HIGH & LOW & Forward \\
\hline LOW & HIGH & Inversion \\
\hline HIGH & HIGH & Stop \\
\hline
\end{tabular}

As shown in Table. 3, "IN 1" and "IN 2" can control DC motor by inputting HIGH or LOW from the Raspberry Pi. This means, the rotation direction can be switched by a combination of inputs signals. 
Next, we explain the pin8 which supply power to the motor, $\mathrm{V}_{\mathrm{s}}$. The power supply connected to this pin determines the power supply of the output of the DC motor itself. In this research we don't use batteries to reduce the weight of the drone. $\mathrm{V}_{\mathrm{S}}$ connected with pin 2 of the Raspberry Pi's GPIO to supply 5 [V]. The control power supply terminal $\mathrm{V}_{\text {ref }}$ of the Pin 4 is used for controlling the PWM signal, but it is not used in this time, it is connected to the pin 8 via the $10 \mathrm{k}$ ohm resistor.

The power supply terminal $\mathrm{V}_{\mathrm{CC}}$ of pin 7 is called a logic side power supply and it is a power supply to this motor driver, which also supplied 5 [V] from pin 2 . In this paper, we only need to use forward operation, so we set output pin IN 1 to HIGH and IN 2 to LOW.

\section{System Configuration}

The flying of drone control from dedicated application shown as Figure 7. The running of drone control from Raspberry Pi. The circuit of the motor car is shown in Figure 8

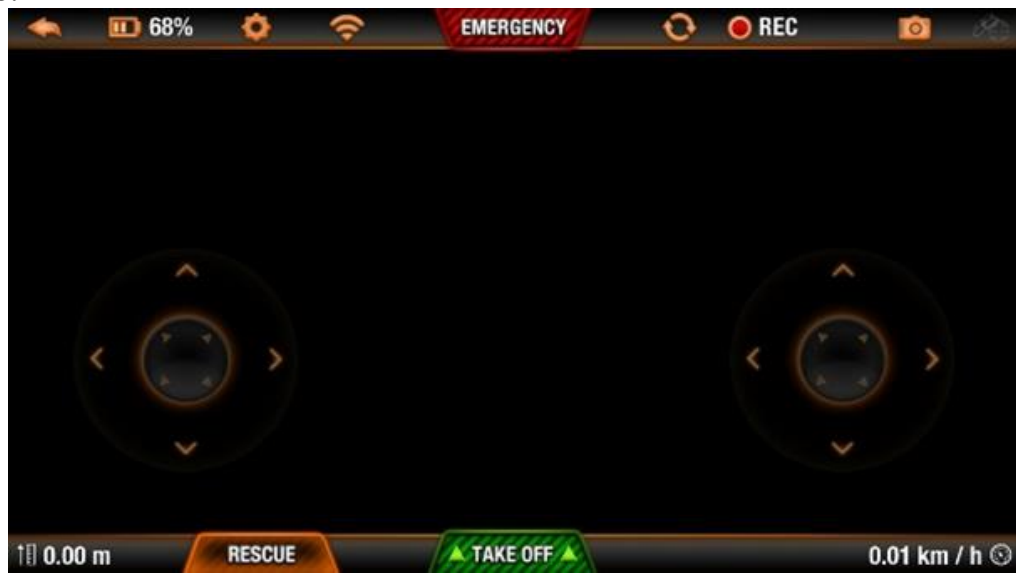

Fig. 7. Official application of AR.drone operation.

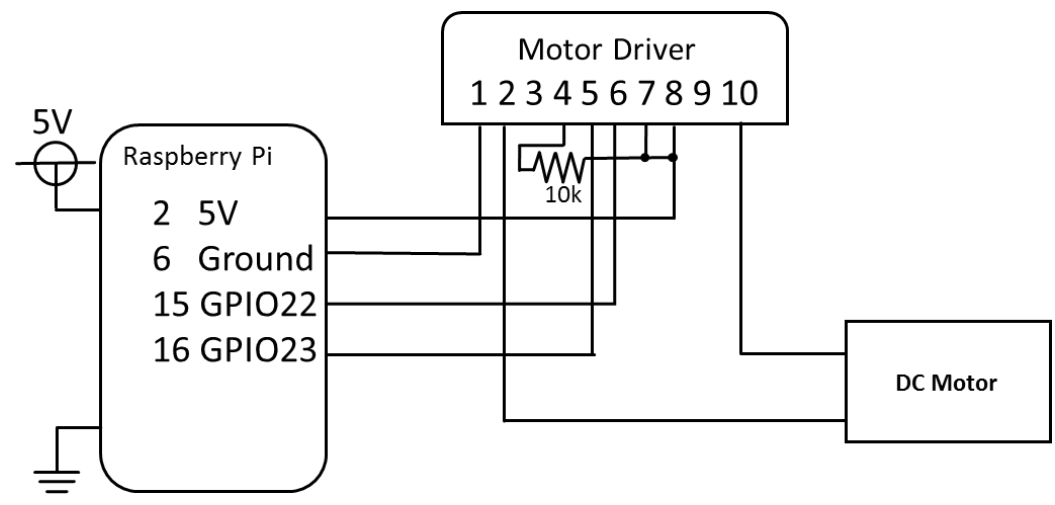


Fig. 8. Circuit of the motor car

Make the circuit shown in Figure. 9 for the car's motor control from the Raspberry Pi's GPIO pin signal. The four GPIO pins are used, pin 5 which is a 5 [V] terminal, Pin 6 which is Ground, Pin 15 and Pin 16 which are general purpose input / output terminals.

Wi-Fi adapter plugged into Raspberry Pi to connect Wi-Fi from drone. A laptop computer for handling car also connects to the same Wi-Fi. This means that we place Raspberry $\mathrm{Pi}$ and laptop on the same network. And, using an application called WebIOPi, link drone, Raspberry Pi (tire type car) and laptop computer. WebIOPi is an application for accessing Raspberry Pi GPIO from browser [8]. Using this, we access the GPIO pins of Raspberry Pi from the browser of the laptop computer and control the tire type car. The flowchart of the motor car is shown in Figure 9. The whole system is shown in Figure 10.

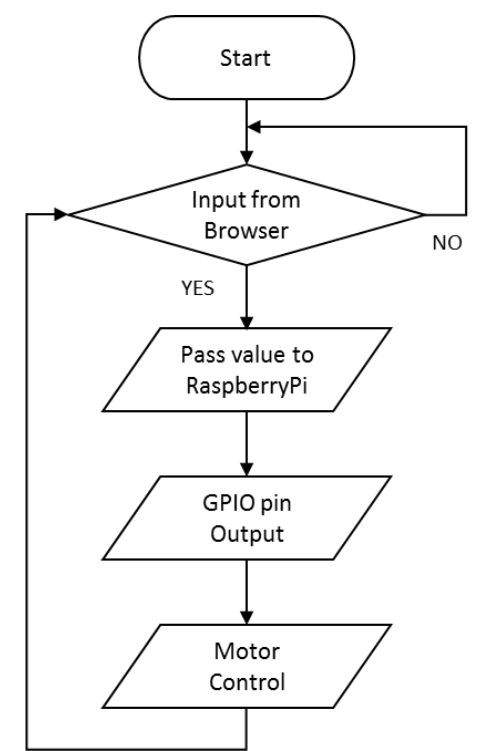

Fig. 9. Flowchart of the control motor car.

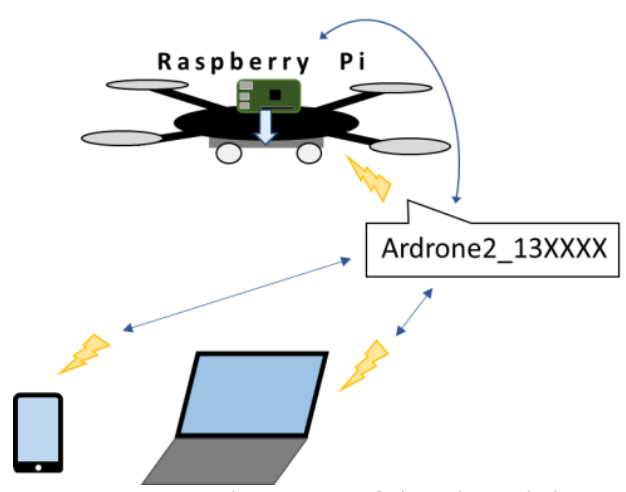

Fig. 10. Control system of developed drone. 


\section{Experiment}

\subsection{Measuring Method}

In this research, we develop a drone, and calculate the power consumption of each case in the case of flight and running, and confirm the difference. Specifically, we use a drone with a tire type car that can be operated remotely, and research in a room with less environmental change. About flight, we measure total flight time against total battery consumption. Then, the current value is calculated from the relational the capacity of the battery and the power consumption. About running, we connect resistors in the middle of the wire connecting between the positive electrode of the battery and the drone. Then, we measure the voltage applied to the resistance, and obtain the current value. Then, the power consumption is calculated.

\subsection{Measurement Result}

First, we measure the power consumption during flight, and confirm the difference in power consumption before installing the system and after doing it. $W$ is calculated from the formula (1), (2), battery capacity $C(1500[\mathrm{mAh}])$, the total flight time $t[\mathrm{~s}]$ and output voltage $V(12.3[\mathrm{~V}])$. The speed is $0.1[\mathrm{~m} / \mathrm{s}]$.

$$
\begin{gathered}
\mathrm{I}=\mathrm{C} /(\mathrm{t} / 3600) \\
\mathrm{W}=\mathrm{I} \times \mathrm{V}
\end{gathered}
$$

Table. 4. Flight experiment

\begin{tabular}{|c|c|c|}
\hline System & Flight Time(s) & Consumption(W) \\
\hline Before & 630 & 105 \\
\hline After & 625 & 106 \\
\hline
\end{tabular}

Table. 5. Experimental results.

\begin{tabular}{|c|c|}
\hline Times & Current $(\mathrm{mA})$ \\
\hline 1 & 442 \\
\hline 2 & 447 \\
\hline 3 & 443 \\
\hline 4 & 447 \\
\hline 5 & 446 \\
\hline 6 & 442 \\
\hline 7 & 442 \\
\hline 8 & 441 \\
\hline 9 & 442 \\
\hline 10 & 445 \\
\hline Average & 443.5 \\
\hline
\end{tabular}


As shown in this table, it is found that the power consumption increased $1[\mathrm{~W}]$ by installation of the system. Next, we research the power consumption of running. We checked current value from the battery during it runs. The average current value is $443.5 \mathrm{~mA}$. Since the output voltage of the battery is $12.3 \mathrm{~V}$, the average power consumption is $5.5 \mathrm{~W}$.

The above results are summarized as follows.

Drone before system installation (flying): $105 \mathrm{~W}$

Drone after installing the system (flying): $106 \mathrm{~W}$

Drone after installing the system (running): $5.5 \mathrm{~W}$

\section{Conclusion}

From the results of the experiment, we find that the power consumption is very low when running the drone car compared with the case of flying. We conclude that using the function of vehicle lead lower power consumption. Furthermore, this result shows that power consumption of the tire type is lower than crawler type. As a future task, we are planning to equip with AI [9-15]. It realize that a system switches autonomously between running and flight according to the environment.

Acknowledgments. This work was supported by JSPS KAKEHI (17K14694), Leading Initiative for Excellent Young Researcher (LEADER) of Ministry of Education, Culture, Sports, Science and Technology-Japan (16809746), Research Fund of State Key Laboratory of Marine Geology in Tongji University (MGK1608), Research Fund of State Key Laboratory of Ocean Engineering in Shanghai Jiaotong University (1510), Research Fund of The Telecommunications Advancement Foundation, Fundamental Research Developing Association for Shipbuilding and Offshore and Strengthening Research Support Project of Kyushu Institute of Technology.

\section{References}

1. Akihito Kobayashi : "The singular impact of drone business $\sim$ A new market opened by small unmanned aircraft ", Asahi Shimbun Publication, 2015, July

2. Ministry of Economy Trade and Industry : "Road map of utilization of small unmanned aircraft and technology development", 2016, April

3. Yomiuri Online : "Examination of the creation of Zero-based Special Zone for drone delivery experiment", 2016, December

4. NTT data, Masato Kawamura, Hiroshi Ootuka, Yuusuke Kobayashi, Takeshi Koyama, Tomoya Miyazaki, Yuuki Ishiguro, Kouhei Kozima : "IoT / Sensor mechanism and utilization seen by picture" 2015

5. Izumi Nakagawa, Junko Takeda, Whiteman Philip : "The aircraft book", 2015

6. Parrot AR. Drone 2.0, https://www.parrot.com/fr/drones/parrot-ardrone-20-elite$\% \mathrm{C} 3 \% \mathrm{~A} 9$ dition\#parrot-ardrone-20-elite-edition

7. Raspberry Pi, https://www.raspberrypi.org/

8. The Raspberry Pi Internet of Things Toolkit, http://webiopi.trouch.com/ 
9. Y. Li, H. Lu, J. Li, X. Li, Y. Li, S. Serikawa, "Underwater image de-scattering and classification by deep neural network," Computers and Electrical Engineering, vol.54, pp.68-77, 2016.

10. S. Mu, C. Zhou, H. Lu, "Neural network based on-line stabilization for humanoid robots," In Proceedings of 2016 International Symposium on Computer, Consumer and Control, pp.140-143, 2016.

11. M. Chen, Y. Hao, M. Qiu, J. Song, D. Wu, I. Humar, "Mobility-aware Caching and Computation Offloading in 5G Ultradense Cellular Networks," Sensors, Vol. 16, No. 7, pp. 974-987, 2016.

12. M. Chen, J. Yang, Y. Hao, S. Mao, K. Hwang, "A 5G Cognitive System for Healthcare," Big Data and Cognitive Computing, Vol. 1, No. 1, DOI:10.3390/bdcc1010002, 2017.

13. M. Chen, X. Shi, Y. Zhang, D. Wu, M. Guizani, "Deep Features Learning for Medical Image Analysis with Convolutional Autoencoder Neural Network," IEEE Trans. Big Data, DOI:10.1109/TBDATA.2017.2717439, 2017.

14. H. Lu, Y. Li, S. Mu, D. Wang, H. Kim, S. Serikawa, "Motor anomaly detection for unmanned aerial vehicles using reinforcement learning," IEEE Internet of Things, 10.1109/JIOT.2017.2737479, pp.1-8, 2017.

15. H. Lu, Y. Li, M. Chen, H. Kim, S. Serikawa, "Brain Intelligence: Go beyond artificial intelligence," Mobile Networks and Application, pp.1-10, 2017. 